\title{
Lancelot et Guinevere: l'amour dans l'oubli
}

\author{
Stephen Steele \\ Simon Fraser University (Bumaby, British Columbia)
}

\begin{abstract}
Ce qui intéresse Lancelot, comme amant, c'est cette manière de s'abandonner à la contemplation de l'aimée. Et, fidele en cela à Guinevere, il n'est pas à son aise ( l'en poise») dans la vie de chevaliers et d'aventures à laquelle il appartient.

Pour Lancelot, à la recherche de Guinevere retenue prisonnière par Méléagant, il y a peu de repos dans la suite des rencontres, pas la moindre chance de s'absenter des occupations et des divertissements du monde arthurien. Existe chaque fois que le chevalier se retire dans ses pensées la possibilité d'une irruption. Ainsi Lancelot, du côté du pont de l'Epée, qui fait une expérience amoureuse, quitte en même temps ou presque le calme de cette expérience: « $S^{\prime}$ ot et si voit et se mervoille» (v. 770 ). Il n'a pas, dans la surprise, peur du chevalier qui fond sur lui avec sa lance; l'inquiétude naît en lui de sentir l'impossibilité de demeurer seul avec l'image de Guinevere qui l'a entraîné jusqu'au gué. Je vais me laisser prendre à cette impossibilité - pour le dire dans la langue de Sartre - d'échapper aux autres.

Oublier jusqu'à l'oubli de soi, sans avoir à remarquer le chemin parcouru, sans avoir à parler, s'effacer sans avoir à revenir, sans se donner à toutes ces tentations (le lit de l'hôtesse ouvert à Lancelot) qui font une vie chevaleresque: est-ce signe de l'amour ou, par-delà des aventures, obligation envers l'Autre? Lancelot fait profession, et avec quelle dévotion, de tout faire, de faire tout ce qu'il faut pour être de nouveau en présence de Guinevere. ${ }^{1}$ Même si cet engagement envers l'aimée doit passer par l'interdit de l'Amour:
\end{abstract}

S'il lait ce qu'Amors li desfant

Et la ou ele vialt antant

(v. 1241-1242).

Coucher avec la demoiselle qui l'héberge pendant son trajet vers Guinevere - comme $s^{\prime} i l$ devait oublier l'éthique de l'amour pour retrouver ce vers quoi l'amour le porte. 
Accéder à l'oubli, c'est d'abord, pour Lancelot, retrouver Guinevere. C'est veiller à ce que nulle autre, telle l'hôtesse, ne vienne troubler le regard qu'il a pour l'aimée:

N'onques ne torne son esgart

Ne devers li ne d'autre part

(v. 1221-1222).

Personne d'autre que l'aimée. Cette fidélité est-elle alors mise en doute par le fait que l'hôtesse vient partager le lit de Lancelot? Le regard du chevalier refuse de se porter sur l'autre-femme. La fidélité apparaît dans le retour en force de l'écart entre la Reine et la demoiselle, l'aimée et l'autre-femme.

L'idée de mettre une seconde femme à la place de l'aimée aura été l'oeuvre des troubadours dès 1070 et tout au long du douzième siècle. Trait structural de la fin'amors, l'autre-femme a pour fonction, explique Jean-Charles Huchet, de «soulager les peines et les souffrances infligées par l'inflexible hauteur de la Dame» (p.46). Et, selon le même auteur, cela donne matière à distinguer entre amor et amar. Nous ne nous y arrêterons pas. Insistons seulement sur le fait que Chrétien de Troyes coupe court au désir des troubadours, trouvant dans l'autre-femme et l'amour sensuel (amar) de quoi refuser. C'est là, sans doute, l'une des originalités de l'état amoureux de Lancelot: il opte pour l'aimée (la Reine lointaine) contre le corps de son prochain.

Résultat: la demoiselle semble échapper à cette position de troisième personne où les troubadours avaient tenté de l'instaurer. En tout cas, un clivage est installé entre, d'une part, la demoiselle qui va jusqu'à partager le lit du chevalier; et d'autre part, la même demoiselle qui rentre dans sa propre chambre avec infiniment plus de compréhension puisqu'il s'agit de mettre Lancelot à l'aise:

En ma chambre couchier m'irai

Et vos an seroiz plus a eise

(v. 1250-1251).

Quand la demoiselle part semble disparaître avec elle l'idée de l'amour sensuel En quoi Chrétien conteste aussi bien les troubadours que ce que Huchet propose d'appeler l'autre-femme. Mais cette contestation n'est pas véritablement radicale. D'un lit à l'autre, la demoisellen'est pas libre de choisir, car nous sommes toujours depuis le début dans la même économie phallocourtoise du désir. Comme si n'avait été retenu que l'univers signifiant des premiers troubadours, ouvrant sur deux possibilités, la Dame et l'autre-femme.

Cependant, si la demoiselle pouvait se substituer à la Reine, l'idée de la substitution prend, chez Chrétien, une nouvelle signification. Car, d'une part, le chevalier est ouvertement désigné, en écho aux troubadours, comme un homme souffrant d'amour. Tandis que, par ailleurs, le désir de rester seul avec sa souffrance prend le dessus chez Lancelot, et ceci enfin avec l'aide de la demoiselle. Qu'est-ce, qu'est-ce encore qu'aider Lancelot, sinon le dispenser de l'obligation de faire l'amour: «Par droit ne vos puis 
demander» (v. 1259). Bien entendu, la parole de la demoiselle procede d'une volonté $\mathrm{d}^{\prime}$ apaisement ${ }^{2}$ («soulager» la souffrance amoureuse du chevalier); mais l'apaisement ici (loin de l'amour sensuel qu'on associe a l'autre-femme) se charge d'une autre signification, à savoir, l'amor ou l'amour vrai.

Allons plus loin dans la formulation de ce qui fait l'essentiel de cette parole. Tout en rendant au chevalier le loisir qu'il cherche, la demoiselle est amenée, en dernière instance, à donner raison à ce que contient le regard de Lancelot: elle y découvre «quelqu'un qui est tout entier l'ami d'autrui» (v. 1264-1265). Rien d'étonnant donc si, pour retrouver l'amie, Lancelot porte son regard au-delà de l'entourage immédiat.

L'amant ne peut venir jusqu'à l'aimée que dans la perspective de cet oubli complet, et en posant les autres comme gêne ou interférence à son regard. Explication juste de l'oeil de Lancelot mais qui, appliquée à la scène célèbre où le chevalier est saisi par la vue du peigne de Guinevere, n'explique pas tout. A côté de l'état affectif de l'amant, se voit un mouvement semblable chez le narrateur, une parole au rythme débridé, accompagnant aussi loin que possible le personnage dans son amour. Si l'on y regarde de près cet amour est ce qui nourrit la parole du narrateur: ça fait beaucoup parler ${ }^{3}$ Plus encore, ces transports d'amour et de désir, quelque merveilleux qu'ils puissent être, $n^{\prime}$ indiquent-ils pas aussi combien le discours amoureux peut être excessif?

Pourtant, s'il est vrai que la joie de Lancelot pousse loin le narrateur, sa parole n'en repose pas moins sur un repère précis. C'est aux «us et costumes,» à la culture du jour, qu'il est fait appel pour comprendre que Lancelot tombe en extase. Rien de tel que le peigne contenant les cheveux de Guinevere pour arracher le chevalier aux délices du monde. Que Lancelot tourne le dos aux saints, refuse les grands remedes («pleüriche et tiriasque,») ne saurait faire problème, tant le narrateur croit les cheveux de Guinevere capables de guérir tous les maux. Faire en sorte que l'objet amoureux n'ait pas de prix parce que sans équivalent...

C'est un bien étrange objet que le narrateur nous présente ainsi, un objet hors-prix: le peigne de Guinevere (substitut de l'aimée), des cheveux de la reine (métonymie génitale?) qui n'ont pourtant rien à voir avec une économie de l'échange ni une économie de la substitution. Comme si la non-équivalence était la seule forme possible du discours amoureux, comme si l'amour ne venait au discours que pour déjouer tous les mots et les registres imaginables. Ce qui fait peut être que l'ensemble du langage peut servir. Seul est banni le dernier mot sur l'amour, cette possibilité de fixer ou d'encadrer l'expérience amoureuse.

Qui veut parler de l'amour n'en dira jamais assez. Du fait même que le langage amoureux est «impossible, inadéquat, immédiatement allusif quand on le voudrait le plus direct» (Kristeva, p. 9), tout mot d'amour est de trop. Que signifie ici «de trop?» Peut-être ceci, précisément, que ce discours de l'amour, ce discours amoureux est interminable, «long» dira le narrateur de Chrétien.

Rien en l'amour, nul aspect de l'aimée, nulle exaltation de l'amant, n'empêche qu'il échappe à la parole. Pas de mot pour concrétiser l'effet des cheveux sur Lancelot. Pas d'éloge pour témoigner de la vérité de l'amour: 
Et por mançongier et por fol

M'an tanra l'en, se voir an dit

(v. 1480-1481).

Tous les registres - religion, chimie, argent — disent l'amour, même s'ils montrent tous combien inadéquate est la référence. C'est aussi dire quelque chose de cette dimension d'exces (et, ajoute Kristeva, «littérature») dont certains diraient que c'est seulement là que l'amour se laisse exprimer.

Et pourtant, la parole du narrateur se révèle soudain a lui comme «longue.»" Il se pose de manière abrupte la question de savoir s'il fallait en finir avec l'écriture sur l'amour: «Et que feroie ge lonc conte» (v. 1495)? Ce n'est plus Lancelot et Guinevere qui sont en cause, c'est le discours amoureux, aussi bien dans ses rapports avec l'économie du roman que dans ses normes d'expression. Ce serait peut-être cela la mise en question du discours dont parle plus récemment Barthes.

Après ce rappel à l'ordre, le narrateur, reprenant, donne à l'expérience d'amour la possibilité de sens et de mesure. C'est sur la base d'une telle possibilité que le narrateur pourra envisager un moyen de condenser le récit. Il renoue ainsi avec l'idée de faire court, et cela, pour «remettre les choses à leur place, ce qui revient à dire: réimplanter la réalité, peut-être pas toute, mais tant soit peu» (Kristeva, p. 17). ${ }^{4}$

Et cil se delite et deporte

Es chevox qu'il a en son saing

(v. 1498-1499).

Il s'agit là de retourner à ce point de l'histoire où le discours amoureux a pris son envol. D'autre part, et en lui-même, ce retour est indication de quelque chose d'autre, la «remise en place» de l'amant, la promesse de dire l'amour avec très peu. La parole du narrateur, c'est cela aussi.

Revenons à Lancelot tout à la «joie» des cheveux de l'aimée et bousculant à nouveau le regard qu'il porte sur le monde. Bref, il n'y a pas pour lui plus grand étonnement que d'apprendre (de la demoiselle!) que le peigne appartenait à «la femme du roi Arthur» (v. 1423). ${ }^{5}$ Ces mots plongent Lancelot dans l'oubli et lui font perdre conscience. Seule la présence de la demoiselle est en mesure de le tirer de son état de choc-et cela parce qu'il y a honte (et peut-être transparence) dans l'abandon de toute maîtrise de soi:

Quant il la vit, s'en ot vergoigne

si li a dit: «Por quel besoigne

Venistes vos ci devant moi?»

(v. 1443-1445).

Même au moment de la plus grande absence, il n'y a pas de moyen de garder la demoiselle à distance. Or s'il y a un deuxième coup, il est là, dans ce risque qui traverse les aventures du chevalier, à savoir le risque de découvrir autrui «dans le champ de sa présence» (Duras, p. 15). 
Le regard du sujet amoureux se meut dès lors sur le plan où le moi est séparé de son être ou, selon Charles Méla, «dépossédé de lui-même et de son nom» (Charrette, p. 16). Lancelot ne traduit que cet oubli de soi qui est le propre de l'amour chez Chrétien. Le cas de Perceval dans Le Conte $d u$ Graal est très significatif de ce point de vue. Il n'y dans le sang sur la neige que le souvenir de Blanchefleur (l'appel de son visage, dirait Levinas) pour séparer Perceval de lui-même:

Et li sanz et la nois ensanble

La fresche color li resanble

Qui est en la face s'amie,

Et panse tant que toz s'oblie

(v. 4133-4136).

L'amour est là. Perceval se retire de la vie immédiate pour faire place au visage de l'aimée. Et ils vont ensemble, amour et oubli, jusqu'à ce que viennent des chevaliers pour arracher l'amant de ses rêveries.

Que serait un amour sans oubli? Quand Chrétien dit oubli, c'est pour signaler (sans négliger tout ce que cet oubli peut provoquer) que c'est ce qui donne a l'amour sa chance.

\section{NOTES}

${ }^{1}$ Pour être reconnu vrai chevalier et, $d$ ' une certaine manière, amant digne de Guinevere, Lancelot doit venir au secours de la demoiselle: «Ne doi mie avoir cuer di lievre/Quant por li en ceste queste» (v. 1100-1101).

${ }^{2}$ Il faut aussi rappeler que Lancelot a assuré la défense de la demoiselle. Dégager le chevalier de sa promesse de faire l'amour apparaî alors comme un contrepoids.

${ }^{3}$ Sur cet aspect de la fin'amors, et entre autres, Jean-Charles Huchet propose le nom «amour discourtois.»

"Nous soulignons. Nouxs posons cette question de condensation non pas au sujet de Freud et de la psychanalyse, auquels l'étude de Kristeva apporte des éclaircissements, et dont nous n'avons pas l'intention d'examiner la valeur «clinique» (Huchet). Nous la posons au sujet du récit proprement dit.

${ }^{5}$ Les paroles de la demoiselle («Par ma foi, sire,/De la femme le roi Artu») auront suffi a donner à la fin'amors sa formulation la plus décisive. Loin d'être contraint par l'institution conjugale, l'amoureux y trouve sa place. «A l'intérieur de la structure de la fin'amors, dit Huchet, il faut être trois pour que s'écrive un sujet» (p. 46). A chaque personnage de ce triange correspond une gamme de fonctions. 


\section{BIBLIOGRAPHHE}

Barthes, Roland. Fragments d'un discours amoureux. Paris: Seuil, 1976.

Chrétien de Troyes. Le cheoalier de la charrette. Ed. Charles Méla. Paris: Librairie Générale Française, 1992. Française, 1990.

. Le Conte du Graal. Ed. Charles Méla. Paris: Librairie Générale

Duras, Marguerite. L'amour. Paris: Gallimard, 1971.

Huchet, Jean-Charles. L'amour discourtois: La «Fin'Amors» chez les premiers troubadours. Toulouse: Privat, 1987.

Kristeva, Julia. Histoires d'amour. Paris: Denoël, 1983. 